\title{
Observational Constraints on the Formation and Evolution of Globular Cluster Systems
}

\author{
Stephen E. Zepf \\ Department of Physics and Astronomy, Michigan State University, East Lansing, MI 48824, \\ USA \\ e-mail: zepf@pa.msu.edu
}

\begin{abstract}
This paper reviews some of the observational properties of globular cluster systems, with a particular focus on those that constrain and inform models of the formation and dynamical evolution of globular cluster systems. I first discuss the observational determination of the globular cluster luminosity and mass function. I show results from new very deep HST data on the M87 globular cluster system, and discuss how these constrain models of evaporation and the dynamical evolution of globular clusters. The second subject of this review is the question of how to account for the observed constancy of the globular cluster mass function with distance from the center of the host galaxy. The problem is that a radial trend is expected for isotropic cluster orbits, and while the orbits are observed to be roughly isotropic, no radial trend in the globular cluster system is observed. I review three extant proposals to account for this, and discuss observations and calculations that might determine which of these is most correct. The final subject is the origin of the very weak mass-radius relation observed for globular clusters. I discuss how this strongly constrains how globular clusters form and evolve. I also note that the only viable current proposal to account for the observed weak mass-radius relation naturally effects the globular cluster mass function, and that these two problems may be closely related.
\end{abstract}

Keywords. globular clusters:general, galaxies:star clusters

\section{What is the Shape of the Globular Cluster Luminosity Function?}

A natural starting point for determining the key physical processes that make the globular cluster luminosity function (GCLF) is to consider the best available observational constraints on the GCLF. The ideal observational sample for determining the GCLF would both very deep to constrain the faint, low-mass end of the cluster population, and have large numbers of clusters to provide adequate statistics, particularly for the rare very bright and very faint globular clusters. The Milky Way globular cluster system meets the first criterion of probing to very faint globular clusters, and has thus provided some of the basic data indicating the roughly lognormal shape of the GCLF, and the location of the turnover magnitude and estimated mass of the distribution. However, the Milky Way has a relatively poor globular cluster system, numbering only about 150 objects. As a result the Galactic GCLF provides very limited statistical power for any test of the behavior of the GCLF, particularly at the low and high mass ends, each of which are only expected to have a few clusters given the total number of Galactic globulars.

One way to overcome the problem of small numbers of globular clusters in the Milky Way is to study a galaxy with a much richer system of globular clusters. M87 is an obvious choice as it has a combination of an exceedingly rich globular cluster system (with almost two orders of magnitude more globular clusters than the Milky Way), and a moderate distance with its location in the Virgo cluster. However, because the distance to M87 is not negligible, to realize its potential for the study of the low-mass end of the GCLF 


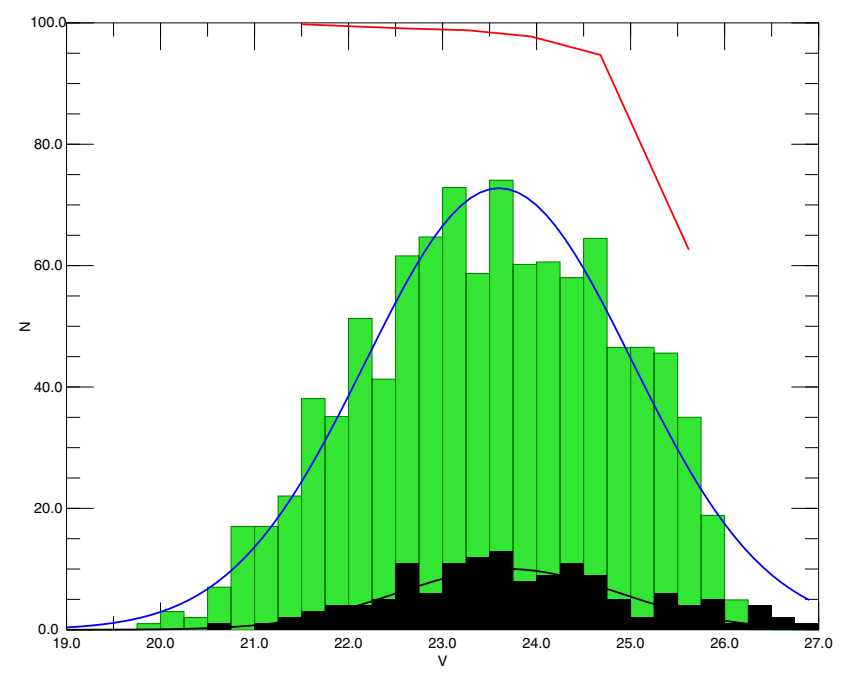

Figure 1. A plot of the globular cluster luminosity function for M87 (upper histogram in green) and the Milky Way (lower histogram in black), with the Milky Way data shifted to the $16 \mathrm{Mpc}$ distance of Virgo. The M87 data is from Waters et al. (2006) and has been completeness corrected, using the completeness function shown as the upper (red) line. Gaussian fits to the M87 and Milky Way data are overplotted on the histograms. The plot makes immediately apparent the dramatic advantage of the much larger numbers of globular clusters in M87 for studying the effects of dynamical evolution on globular cluster systems. As detailed in Waters et al. (2006). the turnover magnitudes of the M87 and Milky Way GCLFs are identical to within the uncertainties, but the M87 GCLF is significantly broader. These conclusions are confirmed by the even deeper 50-orbit ACS dataset (Waters \& Zepf 2008).

requires data that reach to faint apparent magnitudes. Moreover, because the number of background galaxies rises steeply to faint magnitudes, the data must have excellent spatial resolution to distinguish between faint compact galaxies and M87 globular clusters. At brighter magnitudes, ground-based imaging with various image classification and color cuts can create useful globular cluster samples (e.g. Rhode \& Zepf 2001 for a detailed example). However, given the depths required to reach the faint end of the M87 GCLF, and the declining numbers of globular clusters and rising number of galaxies at these faint magnitudes, deep HST imaging is required to reliably probe the shape of the faint end of the GCLF in M87 and other similar galaxies.

A start in this direction was made by various multi-orbit HST WFPC2 studies (e.g. Kundu et al. 1999). These were able to establish that the peaks of the turnover of the GCLF were very similar in M87 and the Milky Way, and also suggested that the width of the M87 GCLF might be larger than that of the Milky Way. However, to accurately test the shape of the GCLF to very faint and low-mass globular clusters required yet deeper data. Until recently, such very deep HST data to probe the faint end of the GCLF were not available. This situation has changed due to extraordinarily deep imaging of M87 obtained originally for studying microlesning in the Virgo cluster. Two such datasets now exist. The first is a 30 orbit WFPC2 dataset, for which we have published an analysis of the faint end of the GCLF in Waters et al. (2006). The second is a 50 orbit ACS dataset which we are now analyzing. In Fig. 1, we show the GCLF resulting from our analysis of the 30-orbit WFPC2 dataset, presented in Waters et al. (2006). The plot makes immediately apparent the dramatic advantage of the combination of very deep 
HST imaging and the large numbers of globular clusters in M87 compared to the Milky Way for studying the GCLF.

The very deep HST data on the M87 GCLF such as shown in Fig. 1 from Waters et al. (2006) provide two key constraints on models of the dynamical evolution of globular clusters through evaporation. This can be readily seen when writing the mass loss of a globular cluster due to evaporation as $\dot{M}=k M^{\gamma} . k$ is then the mass loss rate for a cluster of given mass $M$, and the exponent $\gamma$ accounts for any dependence of this mass loss rate on cluster mass. In terms of comparison to the data, the mass loss rate is primarily constrained by the location of the turnover of the globular cluster mass function, and the dependence of the mass loss rate on $M$ is constrained by the slope of the globular cluster mass function at low masses. These constraints are mostly insensitive to the initial globular cluster mass function for all extant models of two-body relaxation and evaporation. Clusters more massive than the turnover have experienced very little mass loss as a fraction of their total mass, and the low-mass end of the GCLF is composed of globular clusters which have lost an increasing fraction of their initial mass as one goes to fainter globular clusters, Thus the massive end of the observed GCLF is completely determined by the initial GCLF (and possibly dynamical friction for very centrally located clusters) and the low mass end of the observed GCLF is set almost entirely by mass loss from dynamical evolution. As an aside, we note that the shape around the turnover can be affected in detail by the initial mass function if this function is flat or decreases to lower masses.

We can then compare different theoretical models of mass loss due to two-body relaxation in globular clusters to our extraordinarily deep HST data on the M87 globular cluster system. Fig. 2 plots the results of the comparison of our M87 GCLF from the 30-orbit WFPC2 dataset to several theoretical models as shown in Waters et al. (2006). In detail, three theoretical models are shown, each having a different dependence of the mass loss rate on mass as advocated in recent papers. One of these possibilities is that $\dot{M}=$ a constant (i.e. $\gamma=0$ ), and that the mass loss rate is independent of mass (e.g. Fall \& Zhang 2001 and references therein). Another possibility comes from the detailed N-body simulations of Baumgardt \& Makino (2003, hereafter BM03) for which the mass loss rate is approximately $\dot{M} \propto M^{0.25}$. Finally, Lamers, Gieles, \& Portegies Zwart (2005) found $\dot{M} \propto M^{0.38}$ from a combination of fits of the Baumgardt \& Makino (2003) simulations and analytic arguments. Additionally, Fig. 6 in BM03 indicates that the correct mass to use in this calculation is the current mass of the globular cluster, and that in the BM03 simulations the mass loss rate of a globular cluster is not constant in time, and is described well by the $\dot{M} \propto M^{0.25}$ expression calculated for the current cluster mass. There was some discussion of this point at the meeting, but Fig. 6 from BM03 gives a mass loss rate due to evaporation that changes as the globular cluster mass evolves.

The comparison of the deep M87 data to models in Fig. 2 favors mass-loss which is independent of cluster mass. This may be somewhat surprising in that the most state of the art simulations would seem to suggest some mass dependence. In Waters et al. (2006), we discuss some of the possible resolutions of these differences. One is simply the statistical difference, even in the very deep 30-orbit WFPC2 data is not overwhelming. A second issue noted in Waters et al. (2006) is that the mass-to-light ratio (M/L) of the globular clusters may decrease as low-mass stars are preferentially lost from the cluster as it begins evaporating away. We are carrying out new studies that address these points. Our upcoming work uses a 50-orbit ACS dataset to both reach fainter magnitudes and lower masses and to increase the overall number. We also use the mass-to-light ratio evolution of a globular cluster as a function of its disruption time given in BM03 to 


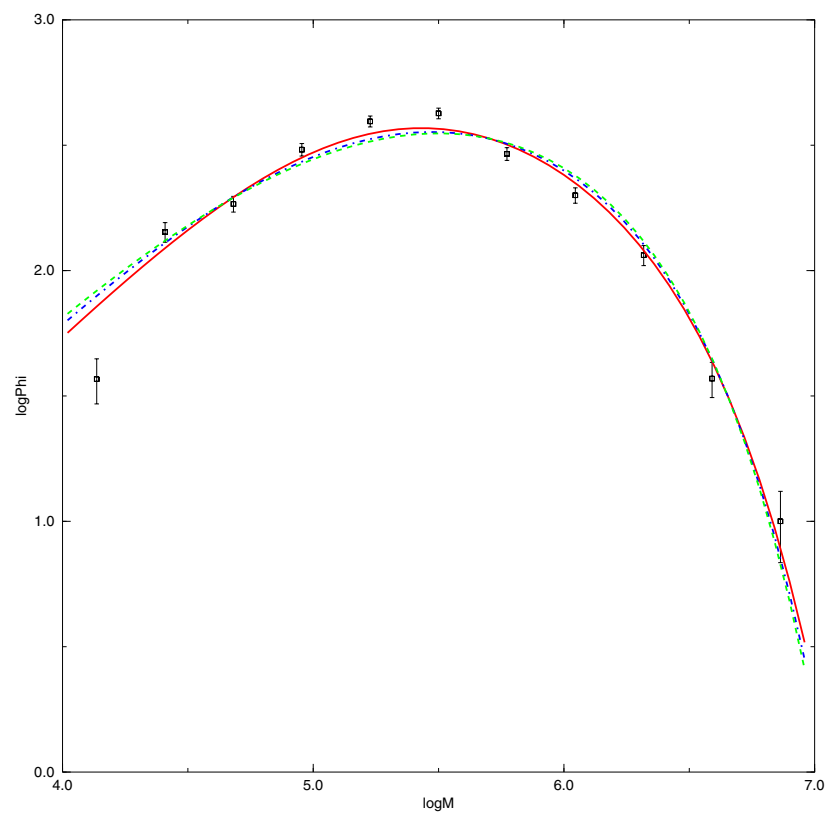

Figure 2. The mass function of the M87 globular cluster system from Waters et al. (2006) analysis of a 30-orbit HST dataset. The three lines are models with different dependencies of the evaporation mass loss rate on cluster mass. The solid line is $\dot{M} \propto M^{0}$, the dot dashed line is $\dot{M} \propto M^{0.25}$, and the dashed line is $\dot{M} \propto M^{0.38}$. The data plotted favor a mass loss rate that is independent of mass, as do new, even deeper data (Waters \& Zepf 2008). This result is driven the by the slope from the turnover to the low-mass end of the mass function, and is not dependent on the initial globular cluster mass function. However, for completeness we adopted a Burkert \& Smith (2000) initial mass function for this plot in order to match the bright end of the globular cluster system.

specifically account for the the changing M/L. The final results from this new work will be published in Waters \& Zepf (2008).

\section{The Unsolved Problem of the Constancy of the GCLF}

Globular clusters closer to the center of a galaxy will have smaller tidal radii for a given cluster mass. This will cause globular clusters closer to the center of a galaxy to lose mass more quickly, and therefore a radial gradient in the globular cluster mass function and GCLF is expected. However, the Galactic GCLF shows no strong changes with radius. This discrepancy between basic theoretical expectation and observation has been noted for some time (e.g. Baumgardt 1998, Vesperini 1997 and references therein). One way around this problem is to have the globular cluster orbits become increasingly radial with distance from the center of the host galaxy, so that all globular clusters have very similar pericenters. Coupled with the assumption that the tidal radii are set at pericenter, one can then recover a constant GCLF with distance from the center of the host galaxy. This scenario was investigated in detail by Fall \& Zhang (2001) who suggested it could account for the lack of an observed radial gradient in GCLFs.

The question is whether the existing globular cluster population has such extremely radial orbits. M87 provides an ideal place to test proposals for the physical origin of the radial constancy of the GCLF. As shown in Vesperini et al. (2003), HST data over 

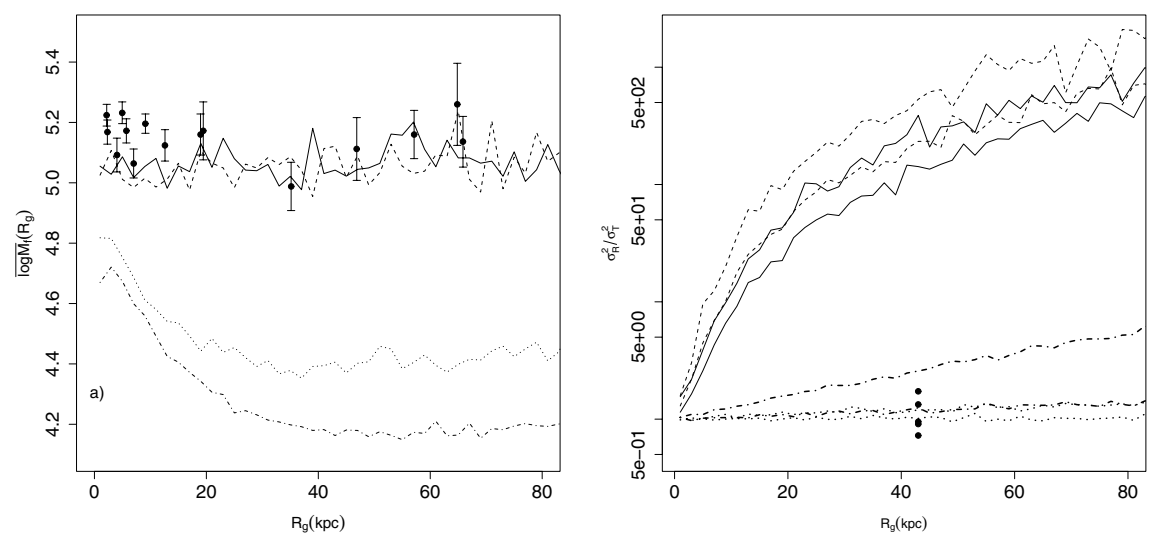

Figure 3. The plot on the left-hand side shows the final globular cluster mean mass vs. projected galactocentric distance for given an initial power-law globular cluster mass function and an initial anisotropy radius equal to 2 (solid line), 3 (dashed line), 15 (dotted line), and $150 \mathrm{kpc}$ (dot-dashed line), from detailed calculations in Vesperini et al. (2003). Large anisotropy radii models are close to isotropic and small anisotropy radii models have orbits which become strongly radial at large galactocentric distances. The constancy of the GCLF with radius is clearly a problem for isotropic orbits. The plot on the right compares the anisotropy of the globular cluster orbits, using the same convention for the models in the plot on the left, to the observed anisotropy of the M87 globular cluster system, which is shown as the points and taken from Romanowsky \& Kochanek 2001). This shows that strongly radial orbits are clearly inconsistent with the observed velocities. Thus, the dilemma that for a power-law initial mass function, evaporation-driven dissolution only produces a constant GCLF with galactocentric distance if the orbits becomes very radial with distance from the galaxy center, but the observed radial velocities rule out such radial orbits (see Vesperini et al. 2003 for details).

a very wide range of radii, from 1 to $75 \mathrm{kpc}$, indicate a generally constant GCLF with galactocentric distance. Other available HST data are consistent with the result, but none yet match this depth and very large radial range. The other key component of the M87 globular cluster system is that several radial velocities studies of large numbers of M87 globular clusters indicate that the current M87 globular cluster population does not have strongly radial orbits (e.g. Côté et al. 2001, Romanowsky \& Kochanek 2001). Vesperini et al. (2003) show directly that their observations of a generally constant GCLF with distance from the galaxy center combined with the results from the radial velocity surveys rule out any model which appeals to strongly radial orbits in the outer regions of galaxies to explain the constancy of the GCLF with galaxy radius (see also Fig. 3).

What is the explanation for the generally constant GCLF seen across the Milky Way and M87? There are three extant proposals, each with its own problems and promise. In order discussed below, these proposals are -1) that hierarchical merging continually mixes the cluster orbits so thoroughly that clusters now located at a wide range of distances from the center of a galaxy, i.e. 2 to $100 \mathrm{kpc}$, have on average had the same local galaxy potential during their lifetime as mentioned in Fall \& Zhang (2001), 2) that early gas expulsion from forming clusters preferentially disrupts lower-mass proto-globular clusters, producing a turnover in the GCLF everywhere at early times (e.g. Baumgardt, Kroupa, \& Parmentier 2008, and references therein), and 3) that globular clusters are formed with an initially power-law mass function, and have a cluster mass-concentration relation such that lower mass clusters have lower concentration and thus are much more likely to be disrupted by stellar mass loss (Vesperini \& Zepf 2003). The difference between 2) and 3) 
is a matter of physical origin and timescale, as 3) occurs on a later timescale in a purely stellar cluster due to stellar mass loss and smaller concentrations for lower-mass clusters, while 2) happens more quickly in a proto-cluster which is still gas-rich.

The appeal of the hierarchical model is that it can work from a power-law initial cluster mass function, as likely found in the Antennae young cluster system (Zhang \& Fall 1999) and consistent with many other young cluster system data. The problem with this proposal is that the complete mixing required to make the GCLF today the same at $2 \mathrm{kpc}$ as $100 \mathrm{kpc}$ has never been demonstrated in any hierarchical model. Mergers are generally found to not mix completely (e.g. White, S.D.M. 1980, Barnes 1988), and a wide variety of observational evidence, such as the presence of metallicity gradients in elliptical galaxies, supports the idea that the most bound material in the progenitors tends to remain the most bound in the merger product. It is also important to note that this thorough mixing must continue until recent epochs. If a population was thoroughly mixed in galactic radius at early times, but was mostly in place at a redshift of one, a radial dependence of the GCLF would be created, as much of the evolution of the cluster would be dominated by its tidal radius at its current location (see Fig. 5 in Vesperini et al. 2003).

Models 2 and 3 which introduce physical processes other than two-body relaxation to help set the scale of the turnover of the globular cluster mass function naturally avoid this problem of the radial constancy of the GCLF. They do not require strongly radial orbits to achieve a constant GCLF, and thus do not violate the observed anisotropy constraints. By far the biggest challenge to these models is the evidence that the mass function for young globular cluster systems is a power-law extending to masses below the current turnover mass observed in old systems. This power-law to lower masses is best established for the Antennae (Zhang \& Fall 1999), but is also consistent with a wide range of other data on young cluster systems. The key question is whether the age of the bulk of the Antennae clusters is older than the age by which these early processes will have disrupted low-mass clusters. As Parmentier \& Gilmore (2007) and others point out, disruption by gas expulsion is not absolutely immediate, but would be expected to take of order of tens of Myr for an unbound cluster to disperse itself into the field. The bulk of the Anntenae cluster sample ranges up to about $100 \mathrm{Myr}$, so this is somewhat older, although it can be argued whether this difference is large enough to be fatal for the model.

The hypothesis that the low-mass clusters are disrupted somewhat later, due to stellar mass loss after they are purely stellar systems (Vesperini \& Zepf 2003), has an important advantage in comparison with the Antennae data that it happens later than the proposed gas expulsion. One key here is establishing whether the globular cluster massconcentration relation observed for the Milky Way globular clusters is primordial. If it is, it seems unavoidable that stellar mass loss will cause some preferential dissolution of low-mass globular clusters, and cause the globular cluster mass function to flatten or possibly turnover at lower masses (Vesperini \& Zepf 2003 and discussion therein). Another key test of the mechanism by which most low-mass globular clusters are destroyed is to study intermediate-aged globular cluster systems, as there is no doubt these are old enough any early destruction mechanism should have changed their mass functions. To date this has proved challenging. Although deep optical data alone may not be able to distinguish intermediate and old globular cluster systems, results suggestive of a powerlaw mass function below the turnoff mass in intermediate-age systems have been found by Paul Goudfrooij and collaborators (e.g. Goudfrooij et al. 2007). One way forward is deep HST near-infrared imaging like that obtained for the intermediate-age system in 
NGC 4365 (Kundu et al. 2005) which allows for a clean enough separation of globular cluster sub-populations to test the dependence of the mass function on age.

\section{The Implications of the Weak Mass-Radius Relationship for Globular}

The masses and radii of globular clusters are for the most part uncorrelated, with only a very shallow relation between the two. This has been established both in the Milky Way (e.g. van den Bergh et al. 1991, Djorgovski \& Meylan 1994, Ashamn \& Zepf 1998), and for extragalactic cluster systems (e.g. Waters et al. 2006, Jordán et al. 2005). This absent or very shallow mass-radius relation stands in contrast to nearby every other type of astronomical object to which globular clusters might be compared. For example, galaxies have a clear mass-radius relation, and clusters of galaxies do as well. Perhaps most importantly scaling relations for molecular clouds, the natural progenitors of star clusters, give $R \propto M^{0.5}$. Studies of globular clusters, both Galactic and extragalactic, give a dramatically shallower relation, with the best current constraint probably being the $R \propto M^{0.04}$ found for M87 globulars by Waters et al. (2006).

Furthermore, studies of young globular cluster systems have also found a very weak mass-radius relation, first in NGC 3256 (Zepf et al. 1999), and now in a number of galaxies (Larsen 2004, Scheepmaker et al. 2007). The lack of a mass-radius correlation in both young and old globular cluster systems strongly suggests the reason for its surprising absence is not due to a long-term evolutionary process, but must be closely related to the formation and early evolution of globular clusters.

The question then is how the formation and early evolution of globular cluster produces such a weak mass-radius relation, particularly when the progenitor clouds seem to have a typically strong relation. Ashman \& Zepf (2001) considered many possibilities for physical mechanisms to account for the weak mass-radius relation of globular clusters. Most of these failed, including such standard ideas as a Schmidt law relating star formation efficiency to density. The one solution that works is to adopt a star formation efficiency proportional to the binding energy of the molecular cloud. Because lower mass clusters have less binding energy per unit mass than higher mass clusters, in this case, low mass clusters have a lower star formation efficiency. As a result of this lower star formation efficiency, when the remaining gas is lost from the clusters, low mass clusters will expand more in response to this mass loss.

Note that this is generally true of any proposal in which lower-mass young clusters lose a greater fraction of their mass than higher mass clusters. Lower mass clusters then respond to this greater mass loss by expanding more than higher mass clusters, assuming the mass loss happens adiabatically. Thus, the radii of low and high-mass clusters become more similar. The same effect might occur in cases in which the cluster loses mass because of stellar mass loss, if more massive globular clusters lose a smaller fraction of their mass.

An invariable outcome of models that successfully produce a weak mass-radius relation is that they flatten the globular cluster mass function (Ashman \& Zepf 2001). Exactly how much they do so depends on the specifics of the model, both how the expansion of a cluster is related to the star formation efficiency, and what the final mass-radius relation is. For example, adopting the final radius of the cluster $r_{f}$ is the initial radius $r_{i}$ divided by the efficiency $\epsilon$, that is $r_{f}=r_{i} / \epsilon$, and the efficiency is proportional to the binding energy per unit mass of the initial system to the power $n$, that is $\epsilon \propto\left(M_{i} / r_{i}\right)^{n}$, then the final slope of the globular cluster system mass function, $\alpha$ is $\alpha=(2 \beta+n) /(n+2)$, where $\beta$ to be the initial slope (see Section 4 of Ashman \& Zepf 2001). If $n$ is around 0.5 , corresponding to a significant but modest mass-radius relation, then the difference 
between the initial and final mass slopes is small, about $10 \%$. If $n$ is large, such as $n=1$ which gives no mass-radius relation, then the difference between the initial and final mass slopes is larger.

Real cluster evolution is undoubtedly more complicated, but these calculations provide an effective framework for showing the connections between the question of the weak mass-radius relation and of the globular cluster mass function. Specifically, the most viable current proposal for the origin of the weakness of the mass-radius relation for globular clusters generically produces a change a flattening of the globular cluster mass function (see Ashman \& Zepf 2001). Whether this flattening is substantial or modest depends on the exact mass-radius relation and to some extent on whether the mass loss and expansion occurs adiabatically. Therefore, one of the obvious observational challenges is to determine the mass-radius relation for globular cluster systems as accurately as possible. A second challenge is to determine the mass function in globular cluster systems that are old enough to have experienced the bulk of their stellar mass loss. If these still have steep power law mass functions like those of molecular clouds, then either the mass-radius relation must be pushed to the limits of the current constraints, or another solution for the weakness of the mass radius relation that does not effect the cluster mass function must be found. The underlying key points are that the data for both young and old globular cluster systems indicate a very weak mass-radius relation, and that extant explanations to produce this have implications for the globular cluster mass function.

\section{Acknowledgements}

Much of the work described above has been carried out in collaboration with Chris Waters, Enrico Vesperini, and Keith Ashman. I gratefully acknowledge support for this work from NSF award AST-0406891 and grants GO-8592 and GO-10543 from the Space Telescope Science Institute.

\section{References}

Ashman, K. M. \& Zepf, S. E. 1998, Globular Custer Systems, (Cambridge University Press) Ashman, K. M., \& Zepf, S. E. 2001, AJ, 122, 1888

Barnes, J. E. 1988, ApJ 331, 699

Baumgardt, H. 1998, A\&A 330, 480

Baumgardt, H., Kroupa, P. \& Parmentier, G 2008, MNRAS, in press

Baumgardt, H. \& Makino, J. 2003, MNRAS 340, 227

Burkert, A. \& Smith, G.H. 2000, ApJ 542, L95

Côté, P., et al. 2001, ApJ 559, 828

Djorgovski, S. \& Meylan, G. 1994, AJ 108, 1292

Fall, S. M. \& Zhang, Q. 2001, ApJ 561, 751

Goudfrooij, P., Schweizer, F., Gilmore, D., \& Whitmore, B. C. 2007, AJ 133, 2737

Jordán, A. et al. 2005 ApJ 634, 1002

Kundu, A., Whitmore, B. C., Sparks, W. B., Macchetto, F. D., Zepf, S. E., \& Ashman, K. M. 1999, ApJ 513, 733

Kundu, A., et al. 2005, ApJ (Letters) 634, 41

Lamers, H. J. G. L. M., Geiles, M., \& Portegies Zwart, S. F. 2005, A\& A 429, 173

Larsen, S. S. 2004, A\&SA 416, 537

Parmentier, G., \& Gilmore, G. 2007, MNRAS 377, 352

Rhode, K. L., \& Zepf, S. E. 2001, AJ 121, 210

Romanowsky, A. J. \& Kochanek, C. S. 2001, ApJ 553, 722

Scheepmaker, R. A., et al. 2007, A\&A 469, 925

van den Bergh, S., Morbey, C., \& Pazder, J. 1991, ApJ 375, 594

Vesperini, E. 1997, MNRAS 287, 915 
Vesperini, E., \& Zepf, S. E. 2003, ApJ (Letters) 587, L97

Vesperini, E., Zepf, S. E., Kundu, A., \& Ashman, K. M. 2003, ApJ 593, 760

Waters, C. Z., Zepf, S. E., Lauer, T. R., Baltz, E. A., Silk, J. 2006, ApJ 650, 885

Waters, C. Z., \& Zepf, S. E. 2008, ApJ in preparation

White, S. D. M. 1980, MNRAS 191, 1P

Zhang, Q. \& Fall, S. M. 1999, ApJ 527, L81 